\title{
Psychological Impacts of COVID-19
}

\author{
RA Bhat ${ }^{1 *}$, S Gulzar ${ }^{1}$, A Muhee ${ }^{1}$, MI Yatoo ${ }^{1}$, S Nisa ${ }^{2}$ and M Nisa ${ }^{3}$ \\ ${ }^{1}$ Division of Clinical Veterinary Medicine, Faculty of Veterinary Sciences and Animal \\ Husbandry, Jammu and Kashmir, India \\ ${ }^{2}$ Division of Animal Nutrition, Faculty of Veterinary Sciences and Animal Husbandry, \\ Jammu and Kashmir, India \\ ${ }^{3}$ Division of Animal Reproduction, Gynaecology and Obstetrics, Faculty of Veterinary \\ Sciences and Animal Husbandry, Jammu and Kashmir, India \\ *Corresponding Author: RA Bhat, Division of Clinical Veterinary Medicine, Faculty \\ of Veterinary Sciences and Animal Husbandry, Jammu and Kashmir, India.
}

Received: June 14, 2021

Published: August 09, 2021

(C) All rights are reserved by RA Bhat, et al.

\begin{abstract}
The coronavirus disease outbreak of 2019 (COVID-19) has triggered a global health catastrophe that has had a profound impact on how we view the world and live our daily lives. Given the existing physical reach of the COVID-19 pandemic in the population, long-term socioeconomic and psychological consequences are unavoidable. The rapid rise in worry and anxiety among individuals as a result of the disease's unknown nature is accompanied by necessary but socially disruptive measures such as lockdowns and quarantines. Post-traumatic stress disorder, depression, anxiety, panic disorders, and behavioural problems are among the psychological and psychiatric diseases that might result. Staying away from family, loneliness, disinformation on social media, financial insecurity, and stigmatisation are all risk factors. Children will be challenged primarily by a lack of structure associated with the closing of schools and colleges. Pregnant women are one of the most vulnerable groups during a viral outbreak, and they are at a higher risk of experiencing anxiety, stress, and depression symptoms during the COVID 19 outbreak. Older adults and people of any age with major underlying medical disorders may be at a higher risk of severe disease from COVID-19 according to currently available information and clinical expertise. With increased vulnerability comes an increase in anxiety, panic, and apprehension among the elderly and their family, which has been ongoing but little discussed. An increase in the prevalence rate of COVID-19 and the resulting restrictions leads to an increase in anxiety; thus, promoting public awareness of the condition and giving positive psychological programmes in the media focused at stress management can help society reduce anxiety.
\end{abstract}

Keywords: COVID-19; Stress; Anxiety; Psychological Impact; Post-traumatic Stress Disorder

\section{Introduction}

The novel coronavirus disease that arose at the end of 2019 began endangering the health and lives of millions of people after a few weeks. It's highly contagious and can cause serious respiratory difficulties, and it has already wreaking havoc on governments and public health systems. Coronaviruses, so named due to the outer fringe of envelope proteins resembling crown (corona in Latin), are a family of enveloped RNA viruses [1].The first case of the COVID-19 pandemic in India was reported on 30 January 2020, originating from China [2].

Presentations of COVID-19 have ranged from asymptomatic/ mild symptoms to severe illness and mortality with common symptoms being fever, cough, and shortness of breath and loss of smell. Other symptoms, such as malaise and respiratory distress, have also been described. These symptoms may develop 2 days to 2 weeks following exposure to the virus (CDC Wuhan, 2020). This 
virus causes mild upper respiratory tract infections in humans and is generally harmful to mammals and birds. This virus was thought to infect only animals until the world witnessed a severe acute respiratory syndrome (SARS) outbreak caused by SARS-CoV, 2002 in Guangdong, China [3]. The COVID-19 virus has now been declared a pandemic. The public's knowledge and attitudes are likely to have a significant impact on the degree of adherence to personal preventive measures and ultimately the clinical outcome. The psychological scars that this monster, insidious virus can inflict on humans - especially because of the social distancing measures and disruption of normalcy it brings in its wake - can be just as gruesome as the physical scars.

After the nationwide lockdown that was enforced in India as well as in other countries to encounter the spread of the coronavirus, the people have found themselves caught in varying degrees of quarantine either self-isolation at home or mandated quarantine to prevent infection. A recent review of research published in the Lancet has found that the Quarantine is linked with post-traumatic stress disorder (PTSD) syndrome, confusion and anger and the effects of which can be long lasting [4]. People have been restricted to their homes. This is taking a serious toll on people's health. Previously chock-o-block highways now glow in the sun with no person or vehicle in the sight. Existential anxiety is induced by such a novel, unprecedented situation.

Stress and anxiety can lower immunity, therefore it's important to keep one's mind and emotions in check. The main concern about the psychological impact of corona is that people are feeling anxious, overwhelmed, or helpless about the corona pandemic. These feelings are especially common among people who are immune compromised or at a higher risk for COVID-19 complications [5]. Hawryluck., et al. [6] reported from a study (conducted on 129 people in Canada) that psychological impacts of quarantine during the SARS outbreak showed a high occurrence of psychological distress, post-traumatic stress disorder and depression and also documented there was a strong correlation between the duration of quarantine and the prevalence of PTSD symptoms.

Epidemics and pandemics like COVID 19 are a periodic phenomenon. During such times, people in the community face a variety of obstacles. The effects of these epidemics and pandemics are typically severe, and they can have a negative impact on a population's mental health. The terror and angst related to epidemics and pandemics also influence the behavior of people in the community. Xu., et al. 2020 [7] has documented that anxiety and fear are very prevalent among patients with COVID19.

\section{Impact on children and youth}

For young people, social isolation and disruption of usual school and college routines can be particularly difficult. The greatest impacts felt by young ones stem from school/college/university closures, being in the house with family members, and not getting to see friends and peers. Teenagers are at a point in life when they are mostly invested in social connections and in separating from their parents, and they have distinct developmental demands than adults. So, COVID-19 social distancing necessities have a different emotional impression on them than on adults. Some children, depending on their age and developmental stage, may struggle to comprehend what the pandemic entails and how it affects our world. Studies have suggested that public health emergencies can have many psychological effects on college students like anxiety, fear, and worry [8]. The COVID-19 problem presented the student fraternity with a unique dilemma. With colleges and universities closed, students had no choice but to return home as soon as possible. In most cases, students were required to leave the hostel, while in others, they were permitted to stay with the proper consents. These surprising shifts have caused great distress among students who are already overburdened with exams, research, and other academic activities. College student's anxiety about COVID-19 might have been related because of their concern about their studies [9] and future employment [10]. The student's concern, on the other hand, could have been created by the steadily rising distances between people resulting from quarantine. Anxiety disorders are supposed to be more prone to develop and aggravate in the absence of interpersonal communication [11,12].

The economic impact of the 2020 coronavirus pandemic in India has largely been troublesome as well. As a result, it's a nobrainer to conclude that career prospects are uncertain and grim in the future, crushing the ambitions and aspirations of young people across the country. This causes them to become more stressed. In the current situation, students are concerned about receiving a de- 
gree on time and the manner in which the entire procedure will be carried out; so, there is a tense air of ambiguity surrounding them. Anxiety and menstruation have a long history of association. Sommer, 1978 [13] has documented the relationship between stress and menstrual distress. Uncertainty about living during a pandemic is causing people to become more stressed. The cortisol hormone (stress hormone) lowers the levels of molecules involved in the menstrual cycle's control. Incarcerated women have high rates of menstrual irregularity and amenorrhea and the occurrence may be associated with certain stresses [14]. Apart from oligomenorrhea, researchers believe that stress play a role in development of PCOS (polycystic ovary syndrome) [15] that can further become cause of concern.

\section{Impact on pregnant women}

Pregnancy is a well-known period of intense changes and in some women, pregnancy may increase vulnerability to psychiatric illness viz., depression. A lot of studies have revealed that COVID-19 had a moderate to severe psychological impression on pregnant women [16]. Antenatal depression affects about $10 \%$ of women in developed countries, and the number of pregnant women prescribed antidepressants has increased over the last decade [17]. Furthermore, during the pandemic, isolation, social distancing, and drastic changes in daily life may raise the risk of depression in vulnerable populations like pregnant women. Therefore, it is of paramount importance to assess the psychological impact of the COVID-19 outbreak. Because of their naturally reduced immune system, pregnant women are more susceptible to infections and are typically seen as having a higher risk of serious consequences [18]. Furthermore, due to their greater concern about vertical transmission to their foetus, pregnant women may be more prone to anxiety. Most of the pregnant women had reported high anxiety and psychological issues regarding the vertical transmission of the disease [19]. Previous epidemiological evidences strongly suggest that pregnant women have a higher risk of serious illness and death from viral infections [20] during pandemics such as influenza and ebola $[21,22]$. Consequently, pregnant women are recommended to reduce social contact by social distancing. It is an well-known fact that in some women, pregnancy modifies how the body fights some viral infections. Although there is yet inadequate evidence for coronavirus, pregnant women are recommended to be especially cautious during this pandemic [23]. Due to Covid19 pregnant women are facing a lot of stress and the stress has detrimental effects to women carrying child. Stress in pregnancy can also lead to miscarriage, delivering before the due date (preterm), giving birth to a small baby (low birth weight) [24]. Depression during pregnancy can develop to postpartum depression if it is not treated. Postpartum depression is a dangerous illness that can continue months after a woman gives birth [25]. It can affect newly mother's health and how well they bond with their baby.

\section{Impact on elderly population}

Despite from younger generations, old aged people and patients associated with chronic underlying diseases are considered at high risk under COVID-19 due to their effete immune system and thereby, COVID-19 affects them more severely. In a comparison of COVID-19 induced pneumonia among young-aged and elderly patients, it has been seen that progression of illness and risk of severity of disease is more in older age group [26]. So, deaths are more common among the older age group and those with chronic underlying disease. As a result, the older population may experience increased worry and fear as a result of their age. In contrast to younger generations, senior persons must not only overcome the hurdles posed by the outbreak's complicated psychological environment, but also overcome the disadvantages posed by their relatively weak immune systems. Therefore, we should pay more attention to the mental health of the elderly people as they are at higher risk of experiencing negative thoughts and feelings when faced with a crisis. In a catastrophic situation, such as a flood or a disease outbreak, elderly people may feel confused, helpless or uncertain about what is going to happen to them and their families. They may also find themselves isolated from others, leading to feelings of loneliness. It is thus important to address these feelings, as they can have a negative effect on the physical and mental health of these people. However, when negative feelings and thoughts continue for too long, or cross a certain limit, they can lead to mental health issues. The impacts of the quarantine can be severe, resulting in feelings of isolation, physical separation from loved ones, grief, anxiety, and chronic stress, all of which can have long-term psychological consequences. According to preliminary study, the elderly have a higher frequency of depressive illnesses, complex post-traumatic stress disorder (PTSD), and adjustment reactions [27]. Irritability or anger, lack of interest in daily activities, complaining that they don't know "how to" or "cannot" do things that were once a part of their routine, crying easily or frequently, disturbed sleep, loss of appetite or food refusal, unwillingness to be 
alone, even for short periods of time are all warning signs of mental health problems in the elderly.

\section{Conclusion}

Currently, at the peak of the COVID-19 outbreak, there is no comprehensive information on the disease's psychological effects on people, and people are experiencing extreme negative feelings as a result of school and workplace closures. It has been established that the prevalence of psychological suffering caused by the development of communicable diseases in society is extremely high. Experts warn that the link between COVID-19 and mental health issues is "bi-directional," meaning that such disorders are not just a result of the pandemic, but their failure to be addressed can contribute to the development of COVID-19 infection. During such times, residents in the community face a variety of obstacles. Lack of knowledge often leads to a careless attitude, which can jeopardise one's ability to meet these problems. The effects of these epidemics and pandemics are typically severe, and they can have a negative impact on a population's mental health. In times of crisis, everyone behaves differently. The COVID-19 pandemic has heightened stress levels in many people. Some people may have trouble sleeping, and many of us are experiencing emotions of isolation and loneliness as a result of the need for social distance. Surprisingly, some study reveals that stress, loneliness, and a lack of sleep all weaken our immune systems, making us more susceptible to diseases like COVID-19.

Worry, anxiety, and fear are common in children, and they might include fears that are quite similar to those that adults have, such as a fear of dying, a fear of their family dying, or a fear of what it means to receive medical treatment. During the COVID-19 outbreak, pregnant women are more likely to experience anxiety and sadness. Prenatal growth depends heavily on a healthy psychological state during pregnancy. As a result, we should pay closer attention to the psychological state of pregnant women during the COVID-19 pandemic. The mental health of the elderly, in reality, requires more attention. When severe public health problems occur, society must pay more attention to the elderly, providing them with more compassionate care and psychological therapies.

\section{Disclosure of Interest}

The authors declare that they have no competing interest.

\section{Bibliography}

1. Burrell CJ., et al. "Coronaviruses". Fenner and White's Medical Virology (2017): 437-446.

2. Sahu KK., et al. "India fights back: COVID-19 pandemic". Heart and Lung: The Journal of Cardiopulmonary and Acute Care 49.5 (2020): 446-448.

3. Zhong N., et al. "Epidemiology and cause of severe acute respiratory syndrome (SARS) in Guangdong, People's Republic of China, in February, 2003". The Lancet 362.9393 (2003): 13531358.

4. Deja M., et al. "Social support during intensive care unit stay might improve mental impairment and consequently healthrelated quality of life in survivors of severe acute respiratory distress syndrome". Critical Care 10.5 (2006): R147.

5. Stein MB., et al. "Coronavirus disease 2019 (COVID-19): Psychiatric illness". (2020).

6. Hawryluck L., et al. "SARS control and psychological effects of quarantine, Toronto Canada". Emerging Infectious Diseases 10.7 (2004): 1206-1212.

7. Xu K., et al. "Management of corona virus disease-19 (COVID-19): the Zhejiang experience". Journal of Zhejiang University (medical science) 49.1 (2020).

8. Mei SL., et al. "Psychological investigation of university students in a university in Jilin province". Med-Soc (Berkeley) 24.5 (2011): 84-86.

9. Cornine A. "Reducing nursing student anxiety in the clinical setting: An integrative review". Nursing Education Perspectives 41.4 (2020): 229-234.

10. Wang C., et al. "A novel coronavirus outbreak of global health concern". Lancet 395.10223 (2020): 470-473.

11. Xiao C. "A novel approach of consultation on 2019 novel coronavirus (COVID-19) related psychological and mental problems: structured letter therapy". Psychiatry Investigation 172 (2020): 175-176. 
12. Kmietowicz, Z. "Rules on isolation rooms for suspected covid-19 cases in GP surgeries to be relaxed". BMJ (2020).

13. Sommer B. "Stress and menstrual distress". Journal of Human Stress 4.3 (1978): 5-47.

14. Allsworth JE., et al. "The influence of stress on the menstrual cycle among newly incarcerated women". Women's Health Issues 17.4 (2007): 202-209.

15. Patel A., et al. "Prevalence and predictors of infertility-specific stress in women diagnosed with primary infertility: A clinic-based study". Journal of Human Reproductive Sciences 9 (2016): 28-34.

16. Berthelot N., et al. "Uptrend in distress and psychiatric symptomatology in pregnant women during the coronavirus disease 2019 pandemic". Acta Obstetricia et Gynecologica Scandinavica 99 (2020): 848- 855.

17. Noordam R., et al. "Prescription and indication trends of antidepressant drugs in the Netherlands between 1996 and 2012: a dynamic population based study". European Journal of Clinical Pharmacology 71 (2015): 369-375.

18. Kourtis AP., et al. "Pregnancy and infection". The New England Journal of Medicine 370.23 (2014): 2211-2218.

19. Thapa SB., et al. "Maternal mental health in the time of the COVID-19 pandemic". Acta Obstetricia et Gynecologica Scandinavica 99 (2020): 817-818.

20. Kwon JY., et al. "New insights into the relationship between viral infection and pregnancy complications". American Journal of Reproductive Immunology 71.5 (2014): 387-390.

21. Jamieson DJ., et al. "What obstetrician-gynecologists should know about Ebola: a perspective from the Centers for Disease Control and Prevention". Obstetrics and Gynecology 124.5 (2014): 1005-1010.

22. Price ME., et al. "A prospective study of maternal and fetal outcome in acute Lassa fever infection during pregnancy". British Medical Journal 297.6648 (1988): 584-587.

23. Kiran L. Senior Consultant-Obstetrics and Gynecology.
24. Dunkel-Schetter C and Glynn L. "Stress in pregnancy: empirical evidence and theoretical issues to guide interdisciplinary researchers". In: Contrada R, Baum A, editors. Handbook of stress science: biology, psychology, and health. New York, NY: Springer Publishing Company (2011): 321-343.

25. Stowe ZN and Nemeroff CB. "Women at risk for postpartumonset major depression". American Journal of Obstetrics and Gynecology 173.2 (1995): 639-645.

26. Liu K., et al. "Clinical features of COVID-19 in elderly patients: A comparison with young and middle-aged patients". Journal of Infection (2020).

27. Banerjee D. "How COVID-19 is overwhelming our mental health". Nature India (2020).

\section{Volume 3 Issue 9 September 2021 (C) All rights are reserved by RA Bhat, et al.}

\section{.}

